\title{
IMPACT OF SINGING BOWL PLAYING ON THE BRAIN USING EEG
}

\author{
Dr. Sundharakumar KB ${ }^{1}$, Dr. Bhalaji $\mathrm{N}^{2}$, Rema Devi R K ${ }^{2}$, Dr. Srinivasan $\mathrm{R}^{2}$ \\ ${ }^{1}$ Department of Computer Science and Engineering, Shiv Nadar University, Chennai, India \\ ${ }^{2}$ Department of Information Technology, SSN College of Engineering, India
}

\begin{abstract}
Singing bowl/Himalayan bowl is a type of standing bell played by rubbing its rim with a wooden mallet. They produce a variety of vibrations to reinstate the natural frequencies of unhealthy and out-of-tone parts responsible for thoughts, emotions and behaviours. The electrical impulses released in the brain during the communication of neurons are known as brain waves. In this work, sixty-five trained subjects of various categories play the singing bowl, and their EEG signal values are taken before playing, during the playing of the bowl and after playing. The signals are recorded during the above durations. Various brain signals (delta, theta, low alpha, high alpha, low beta, high beta and gamma), are observed and analysed for this purpose. These waves are ranked for each instance. The contribution of brain waves at each rank before and after playing the bowl are compared to extract the impact of singing bowl playing on the brain.
\end{abstract}

Key words: Singing Bowl, Brain waves, EEG

Cite this Article: Sundharakumar KB, Bhalaji N, Rema Devi R K, Srinivasan R. Impact of Singing Bowl Playing on the Brain using EEG. International Journal of Electrical Engineering and Technology (IJEET). 12(9). 2021, pp. 55-66.

https://iaeme.com/Home/issue/IJEET?Volume $=12 \&$ Issue $=9$

\section{INTRODUCTION}

The Tibetan Bowls are idiophones or standing bells that vibrate and produce rich tones of sound. The resting bells were initially found in China. On playing, they create ripples in air that are caused by the vibrations generated by the unique resonant frequency of the bowl. The Himalayan bowls have been the part of curative and meditative motives for millenniums. The sound produced from the bowls helps to reinstate the natural pulsation of unhealthy and discordant parts of the spirit, soul, and body. The Himalayan bowl therapies are also called energy entrainment that alters mental state by making one frequency to be dominant than others that results in augmentation of relief from pain, stress, and depression. The distinctive resonance produces an optimal frame for getting subtle awareness, creative thought processes, and intuitive responses. The rich tone that rings from these idiophones awaken the potential of auditory perception with more than eardrum. The enigmatic blending of art, science, 
spirituality, and rhythmic healing is the forte of the singing bowls. Tibetan monk, Gyalwa Karmapa said that the sound of Tibetan Bowls produces vibrations of the void, vibrations that manifest the universe.

Nearly 50 varieties of traditional Tibetan singing bowls are recognized, which includes seven or eight fundamental models, each with its distinctive form and resonance. In our project, we take three of such bowls into consideration, namely the Mani bowls, the Manipuri bowls, and the Trapezoid bowls. They are discussed in the sections below.

The Mani Singing Bowls have a powerful body, a loud vibration, and a unique feature. The illustrious thing about these bowls is their uncommon cone-shape. These bowls have considerably thick and levelled bottom with walls that are narrowed inwards towards the mouth of the bowl. The rim of the bowl is broad and turned inwardly, and the bulgy middle part has a greater girth than the mouth or bottom. The Mani bowls date back to the $16^{\text {th }}$ century and are very rare and hard to find. It is shown in figure 1

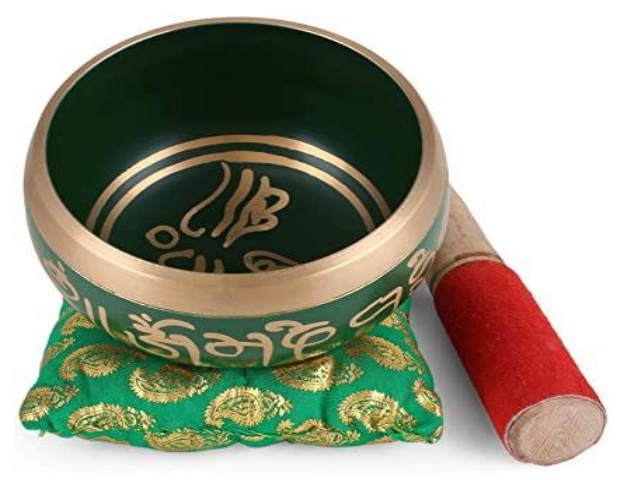

Figure 1 Mani Singing Bowls

Among the Himalayan bowl family, the shallowest ones are the Manipuri model. These bowls can have dense or slim, bare or decorated with a small circular base. The edges of the mouth are finely spread out. The outer lip is often adorned with some inscribed lines as the neckline. Almost all the Manipuri bowls are less than six inches in thickness and have high octave music. It is shown in figure 2

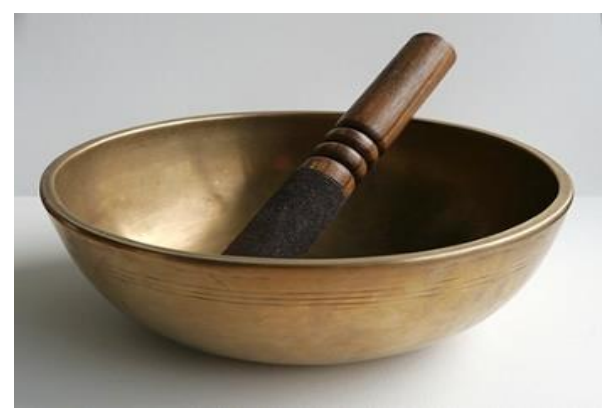

Figure 2 Manipuri Singing Bowls

The Trapezoid singing bowls have two models based on its size; around five inches are the smaller ones and seven to eight inches are the larger ones. The larger bowls are hard to make fine music whereas the smaller bowls have high octaves [4,5]. When seen from the side, the unbending and evenly inclined sides and a levelled base and straight top form a flawless trapezoidal structure. The mouth is wide and faces outwardly with lined impressions. It is shown in figure 3. 


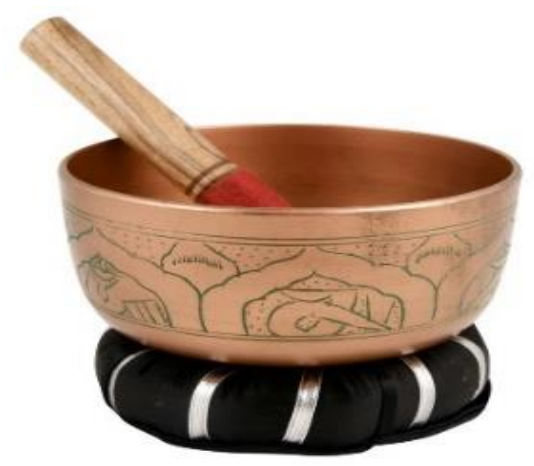

Figure 3 Trapezoidal Singing Bowls

\subsection{Mental Health}

Mental health is the state of inner contentment or an absence of psychological disorders. A person who has an adequate level of emotional and behavioural tuning is said to have stable mental health. If the sound is considered as vibration, then the vibration is heard through each and every part of our body, not by the means of ears alone. One causative for vibrations to restore on a physical plane is due to its capability to renovate on emotional and spiritual levels. Vibrations can heal the inequities on every level of anatomical performance and can have a virtual role in the healing of any medical illness. According to the World Health Organization (WHO), mental health comprises individual well-being, faith in oneself, self-sufficiency, adroitness, intergenerational reliance, and self-discovery of one's logical and emotional capabilities, among others.[11]

\subsection{Brain Waves}

The mass of neurons communicates with each other by generating synchronized electric pulses thereby producing neural oscillations or brainwaves. The speed of neural oscillations is measured in Hertz. The various kinds of brain waves include Infra-low $(<.5 \mathrm{~Hz})$, Delta Waves $(.5$ To $3 \mathrm{~Hz}$ ), Theta Waves (3 To $8 \mathrm{~Hz}$ ), Alpha Waves (8 To $12 \mathrm{~Hz}$ ), Beta Waves (12 To $38 \mathrm{~Hz}$ ) and Gamma Waves (38 To $42 \mathrm{~Hz}$ ). The characteristics of brain waves are shown in fig 1. It is shown in figure 4. To improve neural oscillation sound healing therapy is used.

Table 1 The pattern of brain waves

\begin{tabular}{|c|c|c|c|c|}
\hline Brain Waves & Frequency & Charac & tics & \\
\hline Infra-Low & $<0.5$ & $\begin{array}{l}\text { Brain timing and network } \\
\text { functions }\end{array}$ & \multirow[t]{2}{*}{ Sleep } & \multirow{5}{*}{ 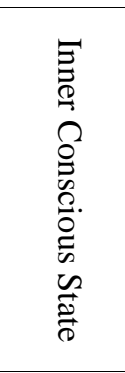 } \\
\hline Delta & $0.5-3$ & Healing and regeneration & & \\
\hline Theta & $3-8$ & Deep meditation & Drowsy & \\
\hline Low Alpha & $8-10$ & $\begin{array}{l}\text { inner-awareness of self, } \\
\text { mind/ body integration, } \\
\text { balance }\end{array}$ & \multirow[t]{2}{*}{ Relaxed } & \\
\hline High Alpha & $10-12$ & $\begin{array}{l}\text { centering, healing, } \\
\text { mind/body connection }\end{array}$ & & \\
\hline Low Beta & $12-18$ & $\begin{array}{l}\text { relaxed yet focused, aware } \\
\text { of self \& surroundings }\end{array}$ & \multirow[t]{2}{*}{ Active } & \multirow{4}{*}{ 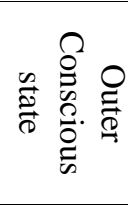 } \\
\hline High beta & $18-30$ & Alertness, agitation & & \\
\hline Low Gamma & $30-70$ & thinking; integrated & \multirow{2}{*}{ Excited } & \\
\hline High Gamma & $70-150$ & thoughts & & \\
\hline
\end{tabular}




\subsection{Sound Healing Therapy}

Sound healing therapy follows the usage of music to enhance external and internal wellness and prosperity. An authorized sound healer can provide such a happening to the patient. This curative vibration includes the sessions like hearing music, getting along with the music with some gestures, performing some musical device, reciting with a musical intonation. The International Academy of Sound healing is involved in providing healing therapy using the Tibetan Singing Bowls. There exist different ways of providing treatment with curative vibrations, which are like involving special music to provoke vibrational thoughts to enhance neuro-oscillations. Sound healing therapy was found to be followed in ancient Greece, where mental illness was treated with special vibrations. Recent research works give more areas where these curative vibrations can be employed like improving immune systems [4] and providing relief from the depression of eleven preterm infants [3]. The different types of sound therapy include Vibroacoustic therapy, guided meditation, Neurologic music therapy, Bonny method, Nordoff-Robbins, Singing bowl therapy, Tuning fork therapy, and Brainwave entrainment Technique [2].

In vibrational therapy, we use singing bowls. The disease can be defined as the appearance of discordance in the body, and inequity in the tissues or body parts. The human being can produce dynamic vibrations and have their frequencies. All the substances can be seen as energy vibrating at different frequencies. The phenomenon of sound healing therapy is that by changing the frequency of vibration, we can change the structure of substances. The vibrations from Himalayan Singing bowls entrain the nervous system of the listener and instigate peaceful and intense meditative states, intelligible mind and instinctive behaviour. The vibrations influence the neurological structures of the human body, fully occupying the relaxation reflex and hindering the depression or discomfort retort. The resonant frequency differs from one part to other parts of the human body. When the frequency of a part is in disharmony or out-of-pitch, that part is said to be affected by or in disease. ${ }^{[12]}$

The Tibetan Bowls utilization in sound therapy is acquired from the fact of considering illness as an interruption in the energy flow of the body. The acoustic vibrations from the bowl reinstate and enhance the energy flow of diseased parts in the body. The founder of stress theory, Dr.Hans Selye studied the stress retort of living beings. He stated that stress is the root cause of health disorders and ailments. His research further conducted by Dr. Herbert Benson, who created the phrase relaxation response. He states this as a physical state of deep rest that changes the physical and emotional responses to stress and the opposite of flight and fight

response. The necessity of relaxation reflex for treating a disease is high, owing to the fact that the nature of stress can cause or worsen the illness.

\section{LITERATURE SURVEY}

Landry's study [8] was made with fifty-one participants who were given two sessions of relaxation for relieving stress. Both the sessions involve a relaxation phase, in which subjects are made to listen to twenty minutes audio. In one session, subjects are asked to rest in silence for twelve minutes before relaxation phase and in other subjects are asked to play the bowl for twenty minutes with an interval of thirty seconds between each strike. The outcome was higher in the session with singing bowl than the one with silence session. The observation showed a change from elevated blood pressure to normal blood pressure and normal heart rates. This study made that employing Tibetan bowls can bring both psychological and physiological enhancements [8].

Tang et al [14] tried to understand whether music healing therapy decreases the negative symptoms of schizophrenia. This study showed that the vibrational healing enhanced the 
patient's ability to communicate with others, increase their social mingling, and to increase their level of interest in external events [14].

This work by Stegemann et al [12] provides an overview of employing music therapy in pediatric care. The study involved various field of application like autism, epilepsy, neonatal care, neuro-rehabilitation, pediatric oncology and palliative care which showed decrease in negative symptoms which are caused due to long term pain. The evidences of involvement of music therapy, music as medicine and other music based activities as a part of treatment has significantly reduced the occurrence of negative symptoms and inducing relaxation response in pediatric sector.

The study conducted by Bradt et al [1] consisted of 52 trials with 3731 cancer patients. The results of this work suggests that music listening may reduce the need for anesthetics and analgesics as well as decrease recovery time and duration of hospitalization. It has also resulted in greater decrease in anxiety and depression.

\section{METHODOLOGY}

The Singing Bowl therapy can be done either as active or receptive music therapy. In active mode, patients are engaged in some form of music-making, by playing the singing bowl. The Receptive mode involves, the patient listening to music played by someone. In our experiment, we chose the active mode. Then the data from the subjects are collected at various levels. The collected data is further analyzed to obtain the results. These works are carried out in two phases (i) Data Collection (ii) Data Analysis.

\subsection{Components}

\section{Different Singing bowls: Mani, Manipuri, Trapezoid bowls}

BrainSense-BCI: To record the brain waves, while playing the singing bowl BrainSense-BCI is used, shown in figure 4. It is a brain computer interface that generates output as 12-bit raw brainwaves with its corresponding brain waves. In traditional method to capture brain waves it requires the subject to apply some gel/ saline material on their head. But, this BrainSense makes it possible without them. The values are given as 8 timestamps for a second. This device has its own interface software named Neuroview and is used to get the values in a comma separated format (CSV) from digital form. In addition, it provides default noise cancellation. It is shown in figure 4

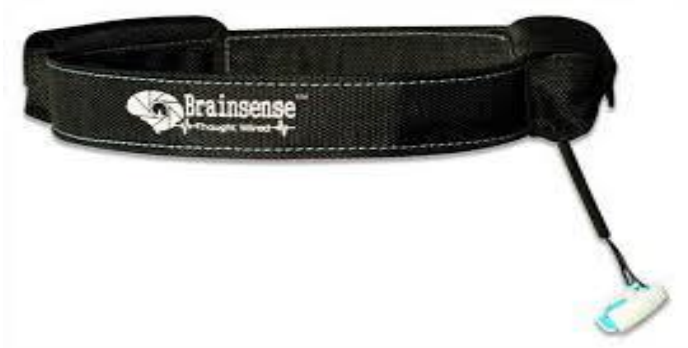

Figure 4 BrainSense-BCI

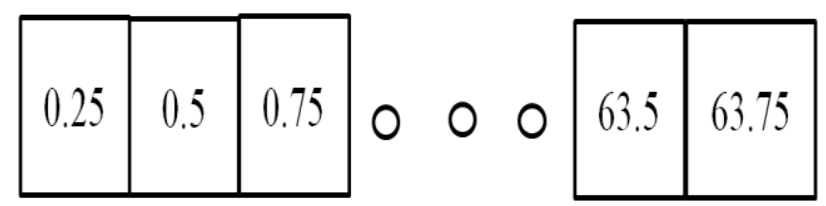

Figure 5 Reading format representation 


\subsection{Experimental Steps}

Step 1 - Training: The sixty-five subjects belonging to different categories are invited to play the bowls. Playing the bowl involves holding the offering-style bowl flat on the palm, perched on the fingertips ${ }^{[8]}$ and rubbing the rim of the bowl using the wooden mallet. Listening to the music or vibration from the bowl and focusing on the area (physical, emotional, mental or spiritual) heals.

Step 2- Recording: To record the EEG signals, given steps are followed.

- The subjects selected are made to sit in an environment without any disturbances.

- Initially, EEG signals are measured using BrainSense- BCI for 60 seconds, without playing the bowl.

- After a resting interval of 5 minutes, another plain reading is taken for 60 seconds.

- Consequent readings of the subject on playing the bowls are taken, after a resting interval of 60 seconds, in similar manner.

- The last reading is taken after playing all the bowls for 60 secondslace figures and tables at the top and bottom of columns.

Step 3 - Analysis: Consider the EEG signals obtained from the subjects. Choose "powerspec.csv" file which has values for various frequency bins $(0-63.75 \mathrm{~Hz})$ is taken for the study. To clean the data, the pre-processing of data is carried out. It is done to check whether eight timestamp values exist for a second. If a second has seven timestamp values and the next second has nine timestamp values then add its first timestamp value as the last value of the previous second. If a second has less than eight timestamps without the above condition, then ignore the entire second data.

- Consider before playing reading of a subject.

- Find the rank of waves for each time instance.

- Find the predominant wave at each rank and form a [1*8] vector.

- Similarly, do for after playing reading.

- Now compare the 2 vectors.

The ranking is done based the contribution of waves at each ranks are taken in descending order.

\section{RESULTS}

The data collected from sixty-five participants have been grouped into various categories based on age, gender, and profession. On categorizing based on age we have six groups with age bin as 10years from 10 to 70 age groups with an average of 10 members each. On the basis of profession, we have four groups namely the educational groups that is the students, the ones who have less manual works that is the white collar jobs, the ones with high physical works that is the physical jobs and the ones who have both physical and mental works that is the job carried by the house wives. The categorization based on gender involves two groups male and female.

In overall analysis, the rank vectors obtained from the before playing readings has Lowalpha waves in Rank 3 which has obtained Rank 5 in after reading. This shows decline of Lowalpha waves. The High-alpha and Low-beta waves have escalated from Rank 4 and Rank 5 to Rank 3 and Rank 4 respectively. The high-alpha corresponds to relaxation and low-beta corresponds to awakening. This implies that calmness is induced in the subjects, when both the waves show significant increase. 


\begin{tabular}{|c|c|c|c|c|}
\hline & BEFORE \\
1 & Delta & & & AFTER \\
\hline 2 & Theta & 1 & Delta \\
\hline 3 & Lalpha & 3 & Theta \\
\hline 4 & Halpha & 4 & Lbeta \\
\hline 5 & Lbeta & 5 & Lalpha \\
\hline 6 & Hbeta & 5 & Hbeta \\
\hline 7 & Lgamma & 6 & Lgamma \\
\hline
\end{tabular}

Figure 6 Change in ranks of overall data

\subsection{Age-based Analysis}

In age-based analysis, the age group 10-20 contributes around $13.84 \%$ of data, 20-30 age group makes $26.15 \%$ of data, 30-40 age group makes $12.30 \%$ of data, $40-50$ age group makes $13.84 \%$ of data, 50-60 age group makes $20.00 \%$ of data, $60-70$ age group makes $13.84 \%$ of data. The subjects belonging to the age groups 10-20 and 20-30 have dropped by one rank in low-alpha, low-beta, high beta, and low-gamma. But there is a significant impact in High-alpha which means that there is an occurrence of healing phase. The change in ranking is shown in figure 7 and figure 8 .

\begin{tabular}{|c|c|c|c|c|}
\hline & BEFORE & & & AFTER \\
\hline 1 & Delta & & 1 & Delta \\
\hline 2 & Theta & & 2 & Theta \\
\hline 3 & $\begin{array}{c}\text { Halpha } \\
\text { Lalpha }\end{array}$ & & 3 & Halpha \\
\hline 4 & Lbeta & 4 & Lalpha \\
\hline 5 & Hbeta & 5 & Lbeta \\
\hline 6 & Lgamma & 6 & Hbeta \\
\hline & & 7 & Lgamma \\
\hline
\end{tabular}

Figure 7 Age:10-20

\begin{tabular}{|c|c|c|c|}
\hline & BEFORE & & AFTER \\
\hline 1 & Delta & 1 & Delta \\
\hline 2 & Theta & 2 & Theta \\
\hline 3 & $\begin{array}{l}\text { Lalpha, } \\
\text { Halpha }\end{array}$ & 3 & Halpha \\
\hline 4 & Lbeta & 4 & Lalpha \\
\hline 5 & Hbeta & 5 & Lbeta \\
\hline 6 & Lgamma & 6 & Hbeta \\
\hline
\end{tabular}

Figure 8 Age:20-30

In the age groups belonging to 30-40, the low-alpha has decreased by one rank and highalpha is increased by one rank. The contribution towards inner-awareness has decreased but has resulted in an inclination towards body/mind healing and regeneration. The change in ranking 
is shown in figure 9. The age group 40-50 has not shown much changes in ranking of data before and after playing the bowl and is represented in the figure 8 .

\begin{tabular}{|c|c|c|c|}
\hline & BEFORE & & AFTER \\
\hline 1 & Delta & 1 & Delta \\
\hline 2 & Theta & 2 & Theta \\
\hline 3 & Lalpha & 3 & Halpha \\
\hline 4 & Halpha & 4 & Lalpha \\
\hline 5 & Lbeta & 5 & Lbeta \\
\hline 6 & Hbeta & 6 & Hbeta \\
\hline 7 & Lgamma & 7 & Lgamma \\
\hline
\end{tabular}

Figure 9 Age 30-40

\begin{tabular}{|c|c|}
\hline & BEFORE \\
\hline 1 & Delta \\
\hline 2 & Theta \\
\hline 3 & Lalpha \\
\hline 4 & Halpha \\
\hline 5 & Lbeta \\
\hline 6 & Hbeta \\
\hline 7 & Lgamma \\
\hline
\end{tabular}

\begin{tabular}{|c|c|}
\hline & AFTER \\
\hline 1 & Delta \\
\hline 2 & Theta \\
\hline 3 & Lalpha \\
\hline 4 & Halpha \\
\hline 5 & Lbeta \\
\hline 6 & Hbeta \\
\hline 7 & Lgamma \\
\hline
\end{tabular}

Figure 10 Age:40-50

The age groups 50-60 and 60-70 has shown similar changes like age group 20-30 rather than having no contribution of high-alpha in the before playing data. This makes clear that laying the bowl has induced a contribution of high-alpha at a predominant rank. So a totally new phase of regeneration or healing has been instigated. The change in ranking is shown in figure 10 and figure 11.

\begin{tabular}{|c|c|c|c|}
\hline & BEFORE & & AFTER \\
\hline 1 & Delta & 1 & Delta \\
\hline 2 & Theta & 2 & Theta \\
\hline 3 & Lalpha & 3 & Halpha \\
\hline 4 & Lbeta & $\begin{array}{lll}4 & \end{array}$ & Lalpha \\
\hline 5 & Hbeta & 5 & Lbeta \\
\hline 6 & Lgamma & 6 & Hbeta \\
\hline
\end{tabular}

Figure 11 Age:50-60 


\begin{tabular}{|c|c|c|c|c|}
\hline & BEFORE & & & AFTER \\
\hline 1 & Delta & & 1 & Delta \\
\hline 2 & Theta & & Theta \\
\hline 3 & Lalpha & 3 & Halpha \\
\hline 4 & Lbeta & 4 & Lalpha \\
\hline 5 & Hbeta & 5 & Lbeta \\
\hline 6 & Lgamma & 6 & Hbeta \\
\hline & & 7 & Lgamma \\
\hline
\end{tabular}

Figure 12 Age 60-70

\subsection{Gender-based analysis}

In gender based analysis, the male group contributes around $32.30 \%$ of data whereas female group holds $67.69 \%$ of data. The male group has a increase in high-alpha rank from rank 4 to rank 5 and decrease in low-beta rank from rank 5 to rank 4 . This implies that the consciousness about the surrounding has been decreased and healing phase has been induced. The changes in rank are shown in figure 13.

\begin{tabular}{|c|c|c|c|}
\hline & BEFORE & & AFTER \\
\hline 1 & Delta & 1 & Delta \\
\hline 2 & Theta & 2 & Theta \\
\hline 3 & Lalpha & 3 & Lalpha \\
\hline 4 & Halpha & 4 & Lbeta \\
\hline 5 & Lbeta & 5 & Halpha \\
\hline 6 & Hbeta & 6 & Hbeta \\
\hline 7 & Lgamma & 7 & Lgamma \\
\hline
\end{tabular}

Figure 13 Male group's rank representation

The female group has shown change in low-beta rank from rank 5 to rank 6 and high beta rank from rank 6 to rank 5. This concludes that the female group was experiencing an agitation mode after playing the bowl. The changes in rank are shown in figure 14 .

\begin{tabular}{|c|c|c|c|}
\hline & BEFORE & & AFTER \\
\hline 1 & Delta & 1 & Delta \\
\hline 2 & Theta & 2 & Theta \\
\hline 3 & Lalpha & 3 & Lalpha \\
\hline 4 & Halpha & 4 & Halpha \\
\hline 5 & Lbeta & 5 & Hbeta \\
\hline 6 & Hbeta & 6 & Lbeta \\
\hline 7 & Lgamma & 7 & Lgamma \\
\hline
\end{tabular}

Figure 14 Female group's rank representation 


\subsection{Profession-based Analysis}

In the profession-based analysis, the student group contributes about $40.00 \%$, the white collar group makes $18.46 \%$, the physical group contributes $23.07 \%$ and the house-wives group makes around $18.46 \%$. The changes in the student group is that the high-alpha has got rank 5 from rank 4 and low-beta has obtained rank 4 from rank 4 in the after playing. It is clear that the student group has experienced a relaxation mode yet with focusing, which can help in their learning. Their agitation has significantly decreased thereby providing focus. The changes in rank are shown in figure 15.

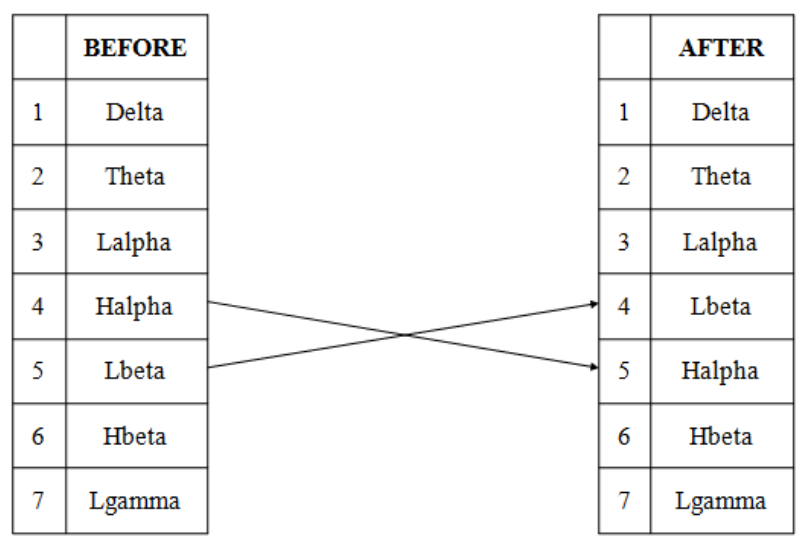

Figure 15 Student group's rank representation

The white collar group has not shown significant changes in the after playing data and its ranking representation is shown in the figure 16.

\begin{tabular}{|c|c|}
\hline & BEFORE \\
\hline 1 & Delta \\
\hline 2 & Theta \\
\hline 3 & Halpha \\
\hline 4 & Lalpha \\
\hline 5 & Lbeta \\
\hline 6 & Hbeta \\
\hline 7 & Lgamma \\
\hline
\end{tabular}

\begin{tabular}{|c|c|}
\hline & AFTER \\
\hline 1 & Delta \\
\hline 2 & Theta \\
\hline 3 & Halpha \\
\hline 4 & Lalpha \\
\hline 5 & Lbeta \\
\hline 6 & Hbeta \\
\hline 7 & Lgamma \\
\hline
\end{tabular}

Figure 16 White-collar group's rank representation

In the physical groups and house-wives group, the low-alpha has decreased by one rank and high-alpha is increased by one rank. The contribution towards inner-awareness has decreased but has resulted in an inclination towards body/mind healing and regeneration. The changes in ranking are shown in the figure 17 and figure 18. 


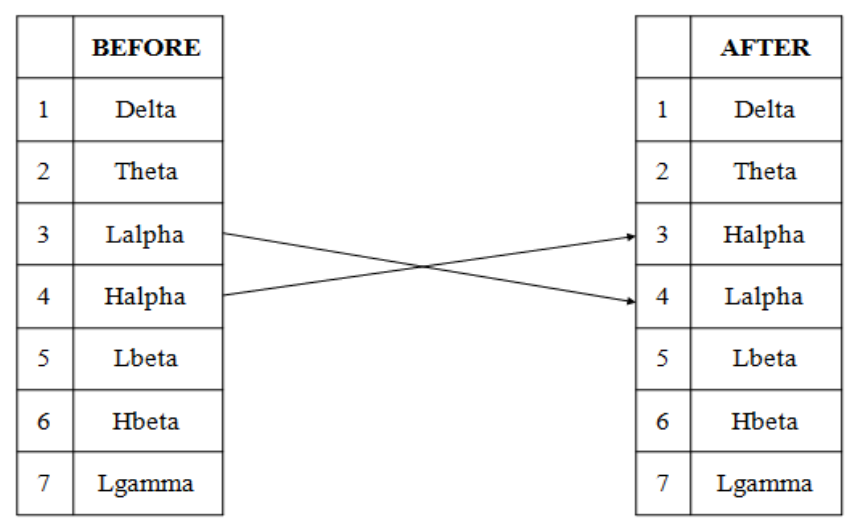

Figure 17 Physical group's rank representation

\begin{tabular}{|c|c|c|c|c|}
\hline & BEFORE \\
\hline 1 & Delta & & & AFTER \\
\hline 2 & Theta & 1 & Delta \\
\hline 3 & Lalpha \\
\hline 4 & Halpha \\
\hline 5 & Lbeta & 3 & Halpha \\
\hline 6 & Hbeta & 4 & Lalpha \\
\hline 7 & Lgamma & 5 & Lbeta \\
\hline & & 6 & Hbeta \\
\hline & & 7 & Lgamma \\
\hline
\end{tabular}

Figure 18 House-wives group's rank representation

\section{REFERENCES}

[1] Bradt J, Dileo C, Magill L, Teague A. Music interventions for improving psychological and physical outcomes in cancer patients. Cochrane Database Syst Rev. 2016;(8):CD006911. Published 2016 Aug 15. doi:10.1002/14651858.CD006911.pub3

[2] Denis Terwagne and John W.M. Bush, Tibetan Singing Bowl Cite, Cornell University Library (2010)

[3] Goldsby, T. L., Goldsby, M. E., McWalters, M., \& Mills, P. J. (2017) Effects of Singing Bowl Sound Meditation on Mood, Tension, and Well-being: An Observational Study Journal of Evidence-Based Complementary \& Alternative Medicine, 22(3), 401-406. https://doi.org/10.1177/2156587216668109

[4] Loewy, Joanne \& Stewart, Kristen \& Dassler, Ann-Marie \& Telsey, Aimee \& Homel, Peter. (2013). The Effects of Music Therapy on Vital Signs, Feeding, and Sleep in Premature Infants. Pediatrics. 131. 10.1542/peds.2012-1367.

[5] Mona Lisa Chanda and Daniel J. Levitin. The neurochemistry of music, Trends in Cognitive Sciences, April 2013, Vol. 17, No.4, CellPress

[6] National Institute for Health and Clinical Excellence (2009). Schizophrenia: Core interventions in the treatment and management of Schizophrenia in primary and 19 secondary cares (update). London: NICE. 
[7] Perry F. Himalyan sound revelations: The complete singing bowl book. London: Polair Publishing, 2014.

[8] Landry, Jayan. (2013). Physiological and Psychological Effects of a Himalayan Singing Bowl in Meditation Practice: A Quantitative Analysis. American journal of health promotion: AJHP. 28. 10.4278/ajhp.121031-ARB-528.

[9] R.M.Aarts ; Okke Ouweltjes and Murtaza bulut An electro-acoustic implementation of Tibetan Bowls: Acoustics and perception, Sage journals VolNo: 45 (2014)

[10] Rolvsjord, R. (2010). Resource-oriented music thersapy in mental health care. Gilsum, NH: Barcelona Publishers.

[11] Suren Shrestha, How to Heal with Singing Bowls Traditional Tibetan Healing Methods, Sentinent Publications (Nov 2013), ISBN 978-1-59181- 230-2

[12] Stegemann T, Geretsegger M, Quoc EP, Riedl H, Smetana M. Music therapy and other musicbased interventions in pediatric health care: an overview. Medicines (Basel) 2019;6(1):25. doi: 10.3390/medicines6010025.

[13] Samantha R. Collin, Chloe L. Keefer, Thomas R. Moore. The Etiology Of Chatter In The Himalayan Singing Bowl, Proceedings of the Third Vienna Talk on Music Acoustics, 16-19 Sept. 2015, University of Music and Performing Arts Vienna

[14] Tang, W., Yao, X., \& Zheng, Z. (1994). Rehabilitative effect of music therapy for residual schizophrenia: A one-month randomised controlled trail in Shanghai. British Journal of Psychiatry, 165 (Suppl.24), 38-44

[15] "The world health report 2001 - Mental Health: New Understanding, New Hope" (PDF). WHO Retrieved 4 May 2014 https://jevondangeli.com/tibetan-singing-bowls-the-ancient-brainentrainment-methodology-for-healing-and-meditation/ 